\title{
Long Non-Coding RNA MALAT1 Decreases the Sensitivity of Resistant Glioblastoma Cell Lines to Temozolomide
}

\author{
Hongwei Lia Xiaoli Yuan ${ }^{\mathrm{b}}$ Dongming Yan ${ }^{\mathrm{a}}$ Dongpeng Lia Fangxia Guan ${ }^{\mathrm{c}}$ \\ Yang Donga Hao Wang ${ }^{a}$ Xianzhi Liua Bo Yanga \\ a Department of Neurosurgery, The First Affiliated Hospital of Zhengzhou University; ${ }^{\text {bDepartment }}$ \\ of Hematology, Henan Provincial Hospital of Zhengzhou University; 'School of Science, Zhengzhou \\ University, Zhengzhou, PR China
}

\section{Key Words}

Glioma $•$ MALAT1 Drug resistance $\cdot$ Epithelial-mesenchymal transition

\begin{abstract}
Background/Aim: Multidrug resistance (MDR) is largely responsible for the failure of chemotherapy. The long non-coding RNA (IncRNA) metastasis-associated lung adenocarcinoma transcript (MALAT1) has been reported to be closely related to tumor biology. In the present study, whether MALAT1 contributes to the resistance of glioblastoma cell lines to temozolomide (TMZ) was investigated. Methods: The glioblastoma cell lines U251 and U87 were exposed to increasing concentrations of TMZ to generate TMZ-resistant colonies (the U251/TMZ and U87/TMZ cell lines). The expression levels of MALAT1 and proteins related to epithelial-mesenchymal transition (EMT) were detected by real-time PCR and western blot, respectively. After the transfection of si-MALAT1 or pCDNA-MALAT1, cell viability, mRNA expression of MDR-associated proteins (MDR1, MRP5 and LRP1), and protein expression of EMT related proteins (ZEB1, Snail and SLUG) were evaluated. Results: The expression of MALAT1 was upregulated in the U251/TMZ and U87/TMZ cell lines compared to that in U251 and U87 cell lines, respectively. The treatment of si-MALAT1 decreased MDR1, MRP5, and LRP1 expression, enhanced cell sensitivity to TMZ, and downregulated ZEB1 protein expression, whereas pCDNA-MALAT1 had the opposite effects. However, the effects of siMALAT1 on MDR -associated protein expression, cell viability, and EMT status were reversed by the transfection of pcDNA-ZEB1, and the effects of pCDNA-MALAT1 were reversed by the transfection of si-ZEB1. In vivo, MALAT1 overexpression enhanced tumors' TMZ resistance and upregulated ZEB1 expression. Conclusion: MALAT1 decreased the sensitivity of resistant glioma cell lines to TMZ by regulating ZEB1.
\end{abstract}

H. Li, X. Yuan and D. Yan contributed equally to this work. 


\section{Introduction}

Glioblastoma is one of the most common malignant brain tumors; it has a poor prognosis and a higher mortality rate in the advanced stage. Chemotherapy is an effective therapeutic option for the treatment of glioblastoma. However, multidrug resistance (MDR) is largely responsible for the modest efficiency of chemotherapy [1]. Suppressing the drug resistance of cancer cells is challenging, but it is a significant step in improving the outcomes of patients with glioblastoma. Temozolomide (TMZ), an alkylating agent, is a first-line drug for the treatment of glioblastoma [2]. It is also used for the treatment of certain other brain tumors. Although oral chemotherapy with TMZ contributes to an overall increase in the survival of glioblastoma patients, the cancer cells eventually develop resistance to TMZ at pharmacotherapeutic concentrations. Its therapeutic efficiency gradually worsens, as with many anti-tumor drugs [3], which leads to the failure of chemotherapy in a large number of patients. Therefore, TMZ resistance is a major obstacle for the treatment of glioblastoma.

The molecular mechanisms of MDR are complex. A series of MDR-associated proteins such as P-glycoprotein 1 (P-gp, also known as MDR1 and ABCB1), multidrug resistance protein (MRP) 1-5, and lung-resistance related protein 1 (LRP1) have been demonstrated to be the critical regulators for MDR [4]. Epithelial-mesenchymal transition (EMT) is a process that governs the conversion of polarized epithelial cells to motile mesenchymal cells, which is very important to gaining the migratory and invasive properties of cancer cells. Loss of E-cadherin, controlled by many transcription factors, is considered to be a fundamental event in EMT [5]. The major characteristic of EMT is the decreased expression of epithelial markers (such as E-cadherin and ZO-1) and the increased expression of mesenchymal markers (such as fibronectin and $\alpha$-SMA). Emerging evidence has demonstrated that EMT is strongly related to MDR in several cancers, and contributes to the pathology of drug resistance [6, 7]. However, the precise mechanisms underlying the effect of EMT on MDR are largely unidentified.

In recent years, long non-coding RNAs (lncRNAs), non-protein coding transcripts that are longer than 200 nucleotides, have been demonstrated to function as regulators of gene expression by epigenetic modification and transcriptional as well as post-transcriptional regulation in various cellular physiological processes and diseases [8,9]. The role of lncRNAs in cancer biology has been widely studied $[10,11]$. Metastasis associated lung adenocarcinoma transcript 1 (MALAT1) was first found in non-small cell lung cancer. Emerging evidence has showed that MALAT1 is implicated in many cancers, including digestive system, gynecological, and urological cancers [12-14]. MALAT1 has also been associated with the clinical outcomes of glioma patients [15], and silencing of MALAT1 leads to the increase of glioma cell apoptosis [16]. It is known that MALAT1 functions as an oncogene partly through inducing EMT. However, the relationship between MALAT1 and MDR remains unclear. In the present study, the role of MALAT1 in TMZ-resistant glioma and the potential mechanisms of MALAT1 were identified.

\section{Materials and Methods}

\section{Cell culture}

Human glioblastoma cell lines U251 and U87 were purchased from the American Type Culture Collection (ATCC, USA) and cultured in Dulbecco's Modified Eagle Medium (DMEM, Gibco, USA) containing $10 \%$ heat-inactivated fetal bovine serum (FBS, Gibco), $100 \mu \mathrm{g} / \mathrm{mL}$ of streptomycin and $100 \mathrm{units} / \mathrm{mL}$ of penicillin at $37^{\circ} \mathrm{C}$ in a humidified atmosphere containing $5 \% \mathrm{CO}_{2}$. The parental U251 and U87 cell lines were exposed to increasing concentrations of TMZ (Sigma-Aldrich, USA) to generate TMZ-resistant colonies (U251/TMZ and U87/TMZ cell lines). In brief, the medium containing $2 \mathrm{ug} / \mathrm{ml} \mathrm{TMZ} \mathrm{was} \mathrm{used} \mathrm{to} \mathrm{culture} \mathrm{the}$ cells for 3 days. A small population of cells survived and passaged. The inductive dose of TMZ was increased by $2 \mathrm{ug} / \mathrm{ml}$ for every generation. The culture was stopped when the final concentration of TMZ reached 20 $\mathrm{ug} / \mathrm{ml}$. The surviving colonies were selected and established as the U251/TMZ and U87/TMZ cell lines. 
Cell transfection

Cell transfection was performed with the Lipofectamine2000 Reagent (Invitrogen, USA) following the manufacturer's protocol. The reference sequences (si-control, si-MALAT1 and si-ZEB1) and overexpressing plasmids (pcDNA, pcDNA-ZEB1, and pcDNA-MALAT1) were synthetized by Shanghai GenePharma Co., Ltd (Shanghai, China). The sequences for siRNAs were as follows: si-control, forward 5'-UUCUCCGAACGUGUCACGUTT-3', reverse 5'-ACGUGACACGUUCGGAGAATT-3'; si-MALAT1, forward 5'-CACAGGGAAAGCGAGUGGUUGGUAATT-3', reverse, 5'-UUACCAACCACUCGCUUUCCCUGUGTT-3'; and siZEB1, forward 5'-GCUGAAAGUCAAGCAAGCAAGCATT-3', reverse 5'-UGCUUGCUUGACUUUCAGCTT-3' .

\section{RNA isolation and real-time PCR}

To determinie the expression levels of MDR1, MRP5, LRP1, and MALAT1, total RNA was isolated and quantitative real-time PCR was performed. In brief, total RNA from the U251, U87, U251/TMZ, and U87/ TMZ cell lines were isolated using the TRIzol reagent (Invitrogen, USA) according to the manufacturer's protocol. After determining the RNA concentration, the complementary DNA (cDNA) was produced by M-MLV Reverse transcriptase (Invitrogen, USA), with RNA acting as a template. Subsequently, real-time PCR was conducted using Power SYBR Green PCR Master Mix (Applied Biosystems, USA) with specific primers. The relative RNA levels were normalized to $\beta$-actin (for MDR1, MRP5, LRP1) and GAPDH (for MALAT1) expression and calculated by the $2^{-\Delta \Delta C t}$ method. Each sample was run in triplicate.

\section{Western blot}

The protein expression levels of ZEB1, Snail, SLUG, E-Cadherin, ZO-1, $\alpha$-SMA, and fibronection were measured using Western blot. Briefly, cells were washed with cold PBS buffer twice and lysed with RIPA buffer (Sigma-Aldrich, USA) containing a protease inhibitor to isolate the total protein. After determination of the protein concentration with the BCA Protein Assay Kit (Pierce Chemicals Co., USA), an equal amount of protein was run on $10 \%$ sodium dodecyl sulfate polyacrylamide gel electrophoresis (SDS-PAGE) and transferred to polyvinylidene fluoride (PVDF) membranes. Subsequently, the membranes were blocked with TBST containing $5 \%$ skimmed milk powder and incubated overnight at $4{ }^{\circ} \mathrm{C}$ with a primary antibody against ZEB1 (1:500, sigma, 3500514, USA), Snail (1:200, Santa-Cruze, 28199, USA), SLUG (1:1000, Abcam, 106077, USA), E-Cadherin (1:1000, Abcam, 133597), ZO-1 (1:200, Abcam, 99462), $\alpha$-SMA (1:1000, Abcam, 21027), Fibronection (1:500, Abcam, 2413), Ki67 (1:5000, Abcam, 92742), PCNA (1:10000, Abcam, 156805), c-caspase 3 (1:200, Abcam, 2302) and $\beta$-actin (1:200, Abcam, 16039). The proteins were then incubated with secondary antibodies conjugated to horseradish peroxidase $(1: 3000, \mathrm{Abcam}, 205718)$ at room temperature for $2 \mathrm{~h}$. Immunoblotting was determined using the ECL kit (Beyotime Biotechnology, China). Each sample was repeated three times.

\section{MTT assay}

The viability of cells after the different treatments was detected by MTT assay. Briefly, the medium was replaced with fresh medium containing $10 \mu \mathrm{L}$ MTT $(0.5 \mathrm{mg} / \mathrm{mL}$, Beyotime Biotechnology, China) for $4 \mathrm{~h}$ of incubation. The MTT solution was removed and the formazan product was dissolved with the supplement of $150 \mu \mathrm{L}$ dimethylsulfoxide (DMSO). The absorbance value at a wavelength of $570 \mathrm{~nm}$ was read using a Microplate reader. Each sample was repeated three times.

\section{Animal experiments}

Forty-eight mice were used in this study. U251 cells were prepared with the stable transfection of lentiviral vector for pcDNA-MALAT1 (LV-MALAT1) to overexpress MALAT1, with the transfection of LVpcDNA-control (LV) acting as the control. Then, a tumor xenotransplant nude mouse model was established with the subcutaneous injection of U251 cells with different treatments. Twenty days after injection, a nude mouse with a tumor size larger than $0.7 \mathrm{~cm}$ was considered to be successful for the establishment of the tumor xenotransplant model. The mice were divided into four groups: 1) mice injected with U251 cells that received normal saline (Control group); 2) mice injected with U251 cells that received the TMZ treatment (TMZ group); 3) mice injected with U251 cells after the LV treatment that were administered of TMZ (LV+TMZ group); and 4) mice injected with U251 cells after the LV-MALAT1 treatment that were administered TMZ (LV-MALAT1+TMZ group). The administration of TMZ $(40 \mathrm{mg} / \mathrm{kg} / \mathrm{d})$ was performed once every three days for six total doses. When the experiment was completed, the mice were sacrificed, and the tumors were removed to measure the tumor volume and determine the ZEB1 expression. The same procedure was repeated using U87 cells. Six mice were in each group. Each sample was repeated three times. 
The animal experiments were approved and performed in accordance with the Animal Care and Use Committee of the First Affiliated Hospital of Zhengzhou University.

\section{Statistical analysis}

In this study, all measurement data were expressed as the mean \pm SEM. An independent sample t-test was used to analyze the difference in the means between two groups. An ANOVA method was performed to compare the differences in the means among three or more groups. Two-tailed $P<0.05$ was considered to be statistically significant. All statistical analyses were conducted with SPSS 18.0 software.

\section{Results}

MALAT1 is upregulated in multidrug-resistant glioblastoma cell lines

To confirm the role of MALAT1 in multidrug-resistant glioblastoma cell lines, real-time PCR and Western blot were explored in the U251/TMZ and U87/TMZ cell lines and their parental cell lines (U251 and U87). The results showed that the mRNA expression levels of drug-resistance related genes (MDR1, MRP5, and LRP1) were significantly upregulated in the U251/TMZ and U87/TMZ cell lines compared with the U251 and U87 cell lines, respectively, confirming the drug-resistant capacity of U251/TMZ and U87/TMZ (Fig. 1A). We observed that the MALAT1 expression level was increased in multidrug-resistant glioblastoma cell lines (Fig. 1B) and that the ZEB1, Snail, and SLUG protein expression levels were also upregulated (Fig. 1C). These data suggested that MALAT1 and ZEB1 might be involved in the MDR of glioma cells.

\section{MALAT1 down-regulation enhances the sensitivity of glioblastoma cells to TMZ}

To determine whether MALAT1 contributes to the development of MDR in glioblastoma cells, the cells were transfected with si-MALAT1 to downregulate its expression and the sensitivity of glioma cells to TMZ was then evaluated. The transfection efficiency of siMALAT1 was confirmed, and the result showed that si-MALAT1 significantly decreased MALAT1 expression in two cell lines (Fig. 2A). As shown in Fig. 2B, the expression levels

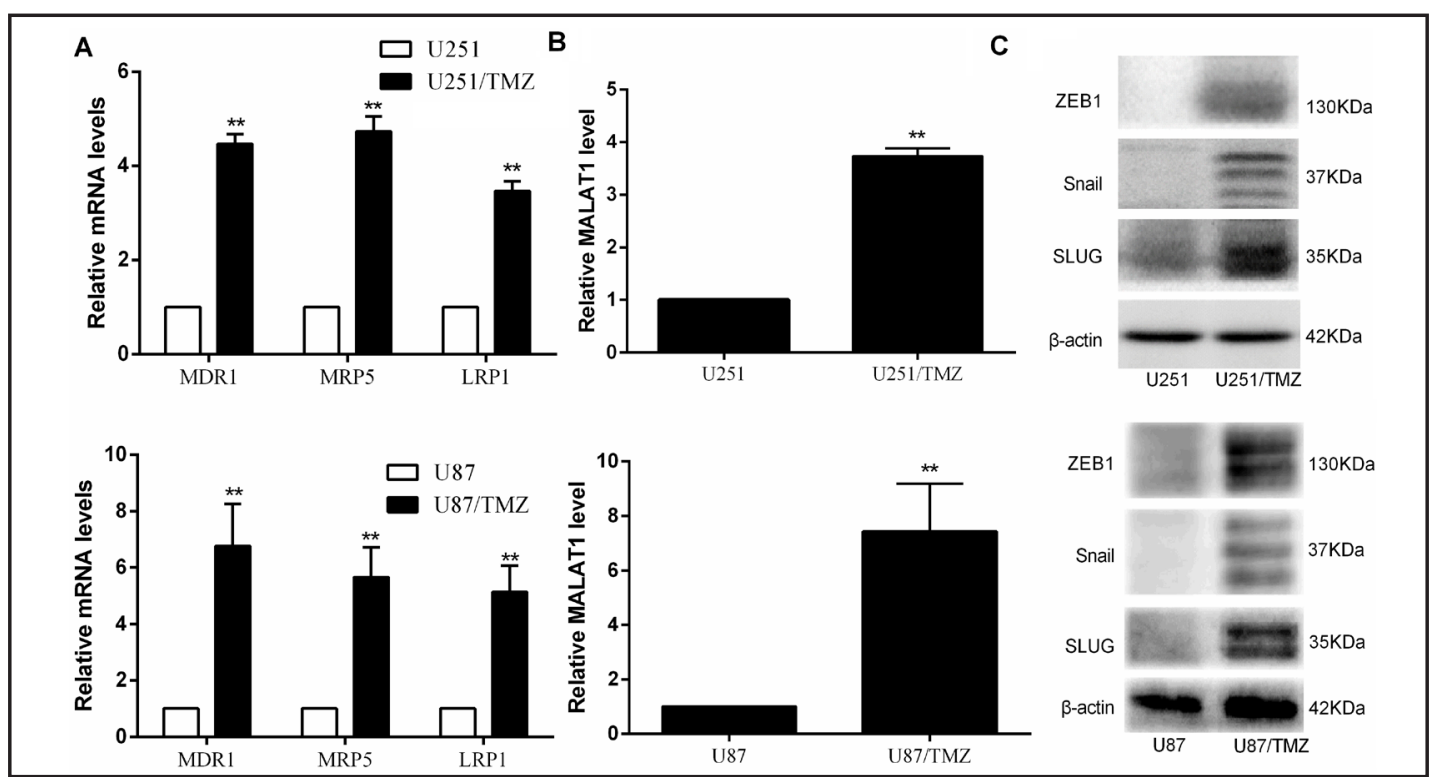

Fig. 1. Expression of MALAT1 and ZEB1 expression in multidrug-resistant glioblastoma cell lines. TMZ: temozolomide. (A) The expression level of drug resistance related genes, MDR1, MRP5, and LRP1, was detected by real-time PCR, with $\beta$-actin acting as a reference gene. (B) The expression level of MALAT1 was measured by real-time PCR, with GAPDH acting as a reference gene. (C) The protein expression of ZEB1, Snail and SLUG was determined by western blot, with $\beta$-actin acting as control. Each sample was repeated three times. The data are shown as mean \pm SEM. ${ }^{* *} P<0.01$, vs. U251 or U87 cell line. 


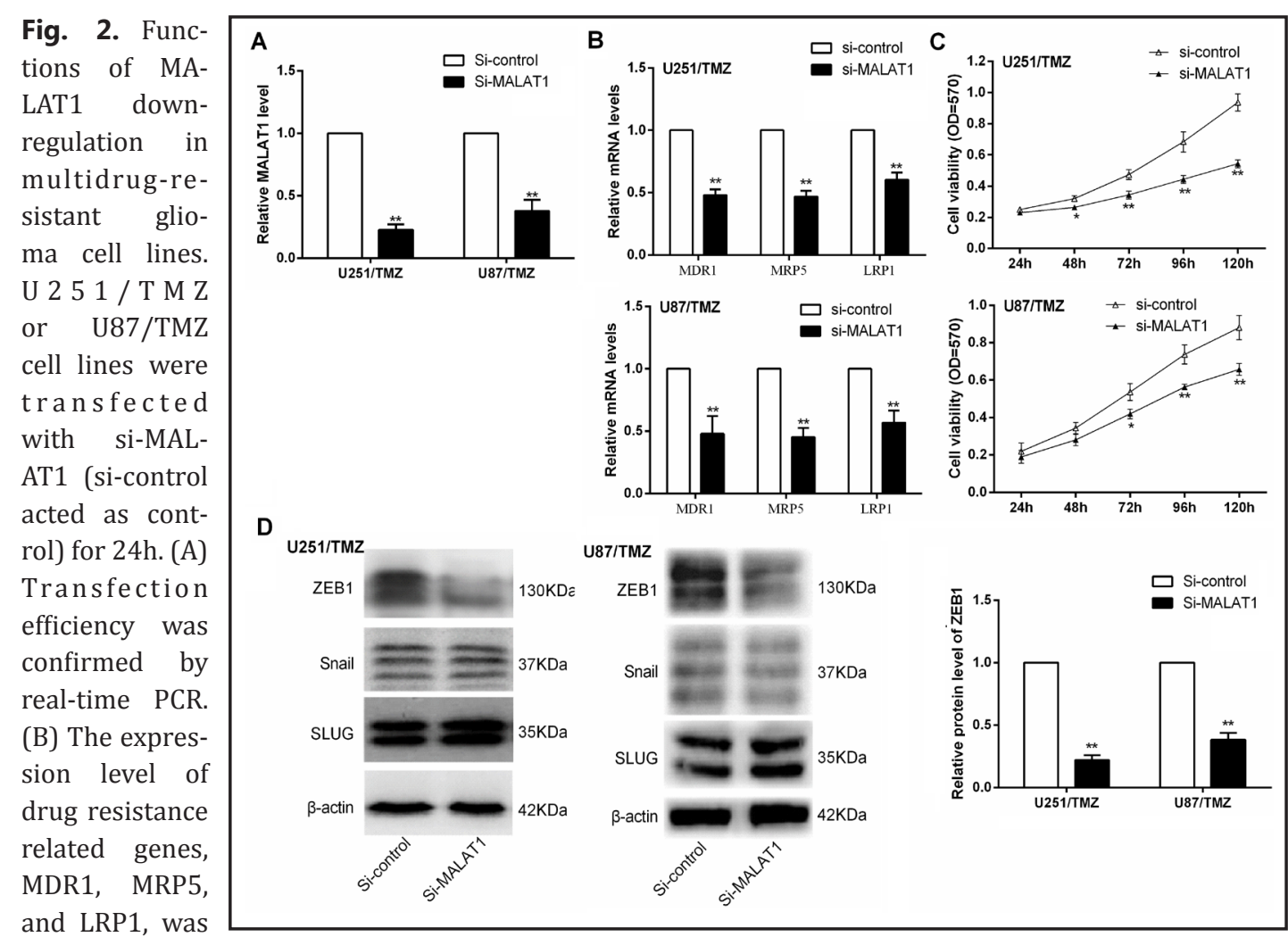

and LRP1, was

detected by real-time PCR, with $\beta$-actin acting as a reference gene. (C) MTT assay was used to detect the cell viability. (D) The protein expression of ZEB1, Snail and SLUG was determined by western blot, with $\beta$-actin acting as control. Each sample was repeated three time. The data are shown as mean $\pm \mathrm{SEM}$. $P<0.05$, ** $P$ $<0.01$, vs. si-control.

of MDR1, MRP5, and LRP1 were decreased by the treatment of si-MALAT1 in U251/TMZ and U87/TMZ cell lines. The cell viabilities of the U251/TMZ and U87/TMZ cell lines were significantly reduced with the transfection of si-MALAT1 (Fig. 2C). We also observed that MALAT1 downregulation contributed to the decrease of ZEB1 protein expression, but did not alter the expression levels of Snail and SLUG (Fig. 2D).

MALAT1 downregulation reduces drug resistance through inhibiting ZEB1 expression

To further investigate the role of MALAT1 in multidrug-resistant glioblastoma and the potential mechanism thereof, the U251/TMZ and U87/TMZ cell lines were transfected with si-MALAT1 or co-transfected with si-MALAT1 and pcDNA-ZEB1. Consistent with the above data, si-MALAT1 led to the downregulation of MDR1, MRP5, and LRP1 expression in mRNA levels as well as a decrease of ZEB1 expression in protein levels, and reduced the cell viability. It was also observed that si-MALAT1 increased the epithelial markers E-Cadherin and ZO- 1 and decreased the mesenchymal markers $\alpha$-SMA and Fibronection. However, the alterations induced by si-MALAT1 were reversed by treatment with pcDNA-ZEB1 (Fig. 3A3C), suggesting that MALAT1 downregulation reduced drug resistance through inhibiting the expression of ZEB1 in glioblastoma cell.

MALAT1 overexpression decreases the sensitivity of glioblastoma cells to TMZ

The effect of MALAT1 overexpression with transfection of pcDNA-MALAT1 on glioblastoma cell sensitivity to TMZ was also investigated. The transfection efficiency of pcDNA-MALAT1 was confirmed and the result showed that pcDNA-MALAT1 significantly increased MALAT1 expression in the U251 and U87 cell lines subjected to TMZ (Fig. 4A). As shown in Fig. 4B, the expression levels of MDR1, MRP5, and LRP1 were increased by the treatment of pcDNA-MALAT1 in two cell lines. The pcDNA-MALAT1 treatment elevated 
Fig. 3. MALAT1 modulates the drug resistance through regulating ZEB1 expression in multidrug-resistant glioma cell lines. Multidrug-resistant glioblastoma cell lines (U251/ TMZ and U87/TMZ) were transfected with si-MALAT1 or co-transfected with si-MALAT1 and pcDNA-ZEB1 for 24h. (A) The expression level of drug resistance related genes, MDR1, MRP5, and LRP1 was detected by real-time PCR, with $\beta$-actin acting as a reference gene. (B) The protein expression of ZEB1, E-Cadherin, ZO-1, $\alpha$-SMA and Fibronection was determined by western blot, with $\beta$-actin acting as cont-
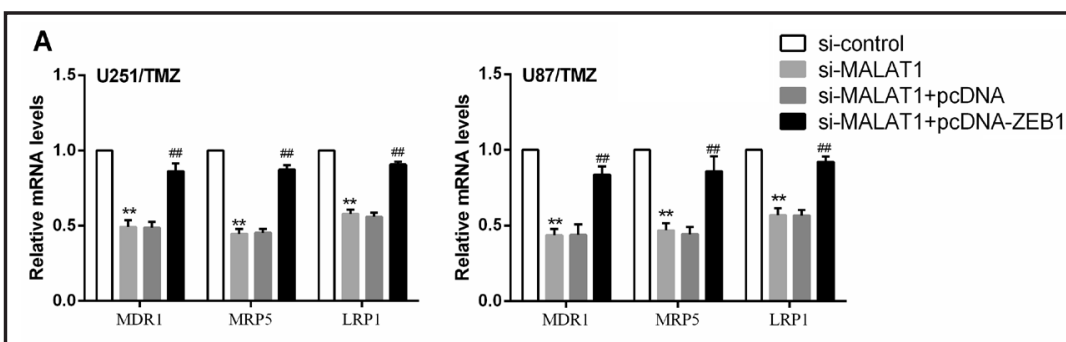

B

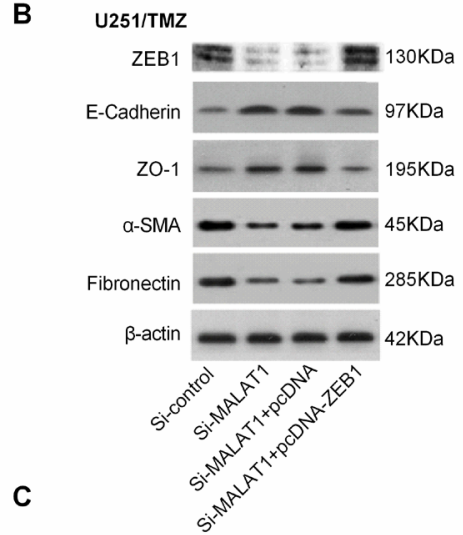

U87/TMZ

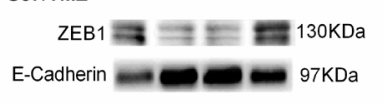

E-Cadherin $=0097 \mathrm{KDa}$
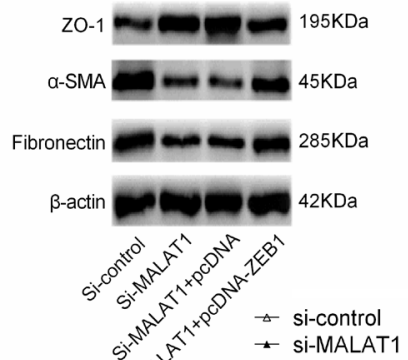

$2 \pi x^{2}+\infty$

- si-MALAT1+pcDNA-ZEB1
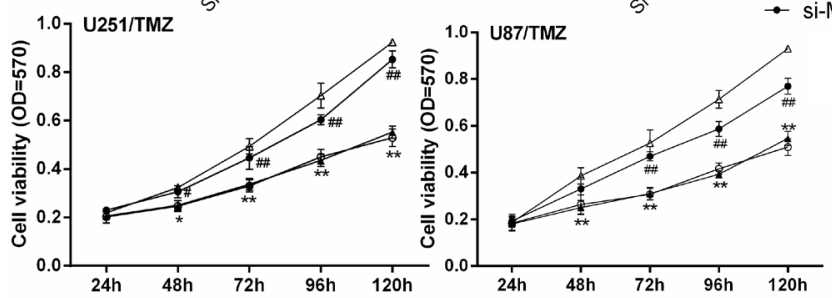

rol. (C) MTT assay was used to detect the cell viability. Each sample was repeated three times. The data are shown as mean \pm SEM. ${ }^{*} P<0.05$, ${ }^{* *} P<0.01$, vs. si-control. \# $P<0.05$, \#\# $P<0.01$, vs. si-MALAT1+pcDNA.

the cell viabilities (Fig. 4C). In addition, ZEB1 protein expression was upregulated by the transfection of pcDNA-MALAT1 (Fig. 4D).

MALAT1 overexpression enhances drug resistance through upregulating ZEB1 expression

The U251 and U87 cell lines subjected to TMZ were subsequently transfected with pcDNA-MALAT1 or co-transfected with pcDNA-MALAT1 and si-ZEB1. The results also demonstrated that pcDNA-MALAT1 downregulated MDR1, MRP5 and LRP1 expression in mRNA levels, reduced the cell viability, decreased E-Cadherin and ZO-1 protein expression, and increased ZEB1, $\alpha$-SMA and Fibronection protein expression. However, the alterations induced by pcDNA-MALAT1 were reversed by the transfection of si-ZEB1 (Fig. 5A-5C). These data suggested that MALAT1 overexpression might enhance drug resistance through upregulating ZEB1 expression in glioblastoma cells.

\section{MALAT1 overexpression enhances tumors' TMZ in vivo}

The function of MALAT1 in the tumor volume in vivo was then discussed. It was demonstrated that TMZ significantly reduced the tumor volume of glioma mice, while the volume was elevated by MALAT1 overexpression with the transfection of LV-MALAT1 in TMZtreated mice (Fig. 6A). TMZ also led to a decrease of Ki67 and PCNA protein expression and an increase of c-caspase-3 protein expression in the tumor tissue of glioma mice, whereas LV-MALAT1 negated this effect. We also found that MALAT1 overexpression increased the expression of ZEB1 protein in glioma mice with the treatment of TMZ (Fig. 6B). These data indicated that MALAT1 overexpression enhanced tumors' TMZ resistance in glioma mice. 

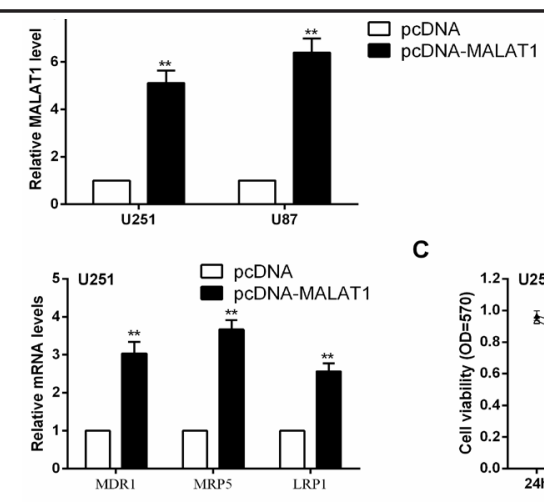

C
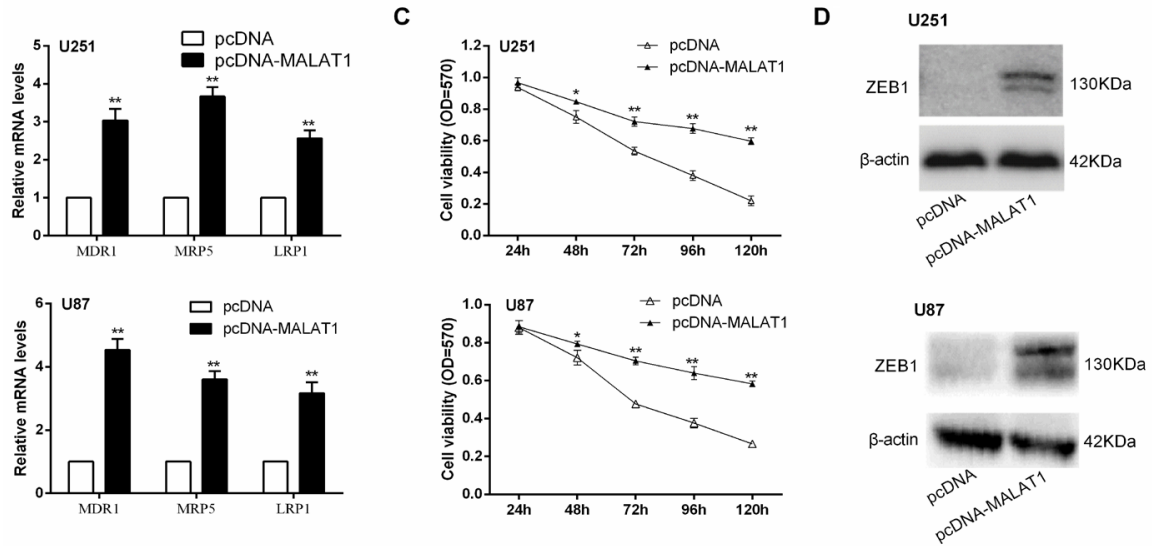

Fig. 4. Functions of MALAT1 overexpression in glioma cell lines. Glioma cell lines (U251 and U87) subjected to $20 \mathrm{ug} / \mathrm{ml}$ were transfected with pcDNA-MALAT1 (pcDNA acted as control) for 24h. (A) Transfection efficiency was confirmed by real-time PCR. (B) The expression level of drug resistance related genes, MDR1, MRP5, and LRP1, was detected by real-time PCR, with $\beta$-actin acting as a reference gene. (C) MTT assay was used to detect the cell viability. (D) The protein expression of ZEB1 was determined by western blot, with $\beta$-actin acting as control. Each sample was repeated three time. The data are shown as mean \pm SEM. ${ }^{*} P<$ $0.05,{ }^{* *} P<0.01$, vs. pcDNA.

Fig. 5. MALAT1 overexpression enhances the drug resistance through upregulating ZEB1 expression in glioma cell lines. Glioma cell lines (U251 and U87) were transfected with pcDNAMALAT1 or co-transfected with pcDNA-MALAT1 and si-ZEB1 for 24h. (A) The expression level of drug resistance related genes, MDR1, MRP5, and LRP1, was detected by real-time PCR, with $\beta$-actin acting as a reference gene. (B) The protein expression of ZEB1, E-Cadherin, ZO-1, $\alpha$-SMA and Fibronection was determined by western blot, with $\beta$-actin acting as control. (C) MTT assay was used to detect the cell viability. Each sample was repeated three time. The data are shown as mean \pm SEM. ${ }^{*} P<0.05$, ${ }^{* *} P<0.01$, vs. pcDNA. \# $P<0.05$, \#\# $P<0.01$, vs. pcDNA-MALAT1+si-control.

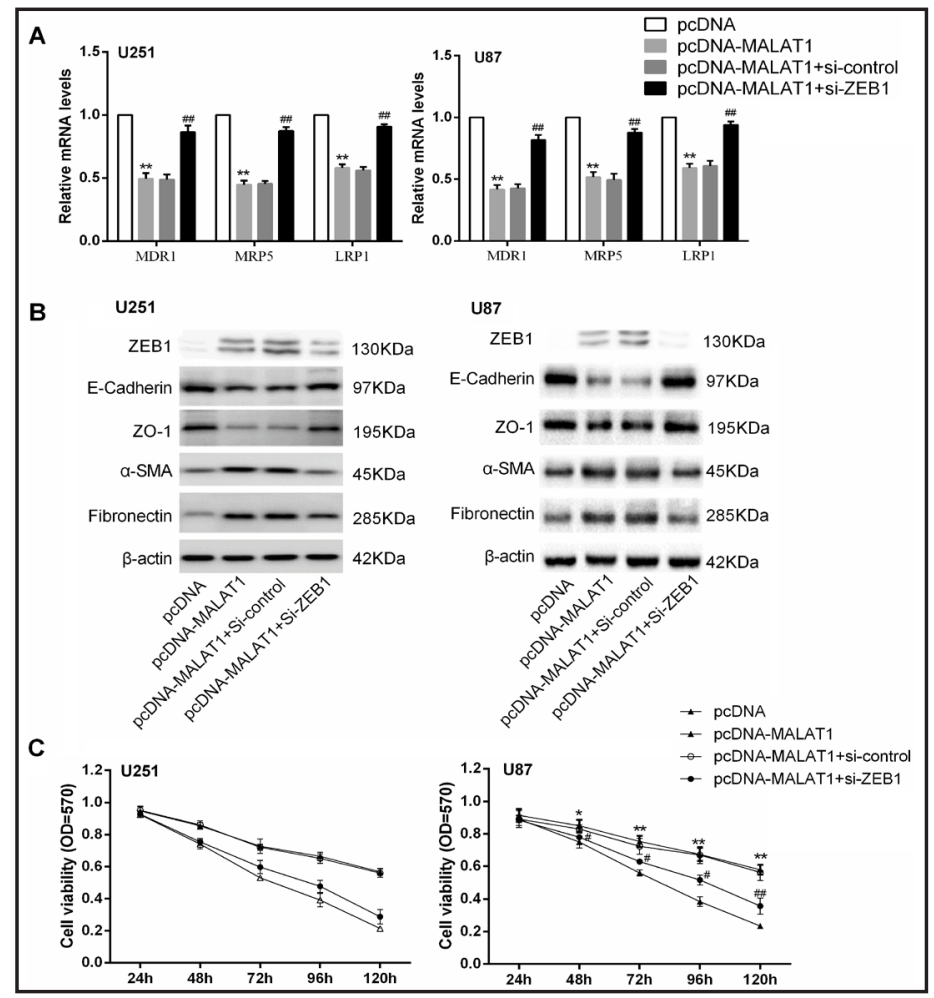

\section{Discussion}

LncRNAs have been demonstrated to play a role in multiple cellular processes relating to tumor biology such as cell proliferation, cell cycle, apoptosis, invasion, and migration, KARGER 
Fig. 6. Function of MALAT1 overexpression in glioma tumor in vivo. TMZ: temozolomide. (A) Tumor volume was measured. (B) Ki67, PCNA, c-caspase 3, ZEB1 protein expression in tumor tissue was determined by western blot. Six nude mice in each group. Each sample was repeated three time. The data are shown as mean \pm SEM. ${ }^{* *} P$ $<0.01$, vs. control. \#\# $P<0.01$, vs. LV+TMZ.

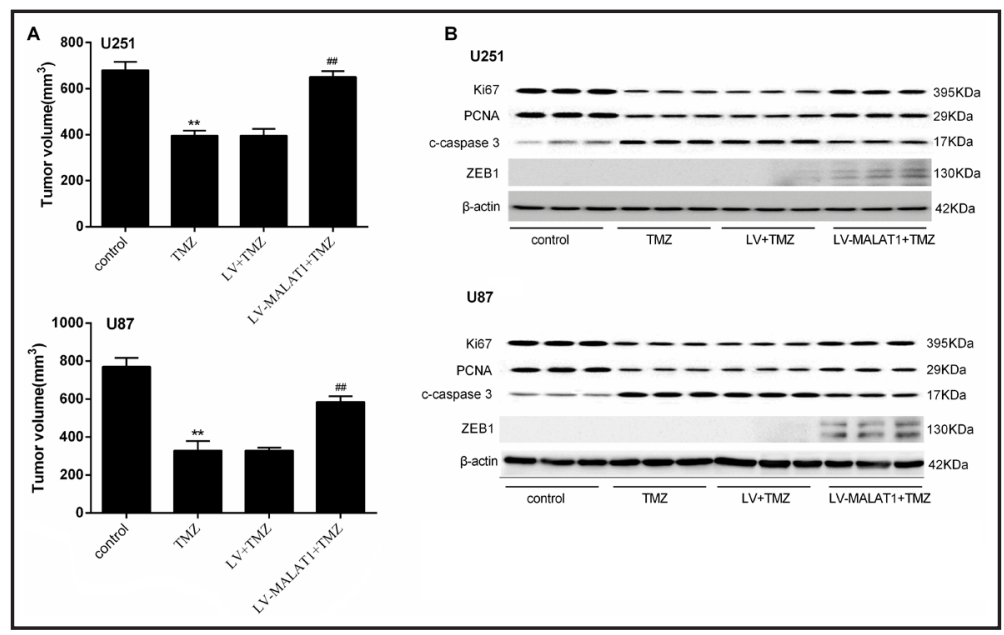

by affecting oncogene expression [17]. Recently, researchers identified some differentially expressed lncRNAs in cancer cell lines and MDR sublines [18, 19]. Pathway analysis showed that 15 pathways corresponded to downregulated transcripts and that that most enriched network was "Alcoholism," while 20 pathways corresponded to upregulated transcripts and the most enriched network was "steroid biosynthesis" [18]. However, the potential mechanism underlying the role of lncRNAs in MDR remains poorly understood.

MALAT1, also known as nuclear-enriched transcript 2 (NEAT2), is expressed in many tissues and widely studied in the development of various cancer types and diseases. There is accumulating evidence that MALAT1 is a prognostic parameter for survival and associated with metastasis, migration and the risk of tumor recurrence after surgical treatment [20]. However, the relationship between MALAT1 and drug resistance in cancer is unknown. In the present study, we found that MALAT1 expression was upregulated in multidrug-resistant glioblastoma cell lines, suggesting that MALAT1 might contribute to the development of MDR. Multiple multidrug resistance-associated proteins that mediate MDR through different mechanisms have been previously identified. MDR1 is a typical ATP-binding cassette (ABC)transporter that pumps foreign substances out of cells; it is responsible for the reduced drug accumulation in cancer cells and for mediating the development of MDR [21]. MRP5, a transporter for cyclic nucleotides, belongs to the subfamily of MRP and functions in the cellular export of its substrate. LRP is a non-ABC transporter and is involved in nucleocytoplasmic transport. All three proteins are confirmed to be critical regulators for MDR [22]. Previous studies have also demonstrated that some lncRNAs, such as HOTAIR, AK126698, UCA1, H19, and UCDR, contribute to drug resistance [23], and Wnt/ $\beta$-catenin, MAPK, p21, apoptosisrelated proteins, and certain other pathways have been proposed to underlie this process $[19,24-26]$. The current study showed that MALAT1 downregulation contributed to the inhibition of cell viability in TMZ-resistant glioblastoma cell lines, indicating a potential role of MALAT1 in the MDR of glioma.

We then investigated the potential mechanism of MALAT1 in TMZ-resistant glioma cell lines. There are diverse factors that result in drug resistance, including genetic and epigenetic changes, alteration of the tumor microenvironment, and EMT. A large body of research has confirmed that EMT is a critical process that is implicated in cancer cell migration and invasion [27]. Recently, the role of EMT in MDR is has been better identified. However, the knowledge of the precise mechanisms in their relationship is very limited. It is well known that the loss of E-cadherin is the fundamental event in EMT and that this loss is induced by several E-cadherin repressors such as Snail, Slug and ZEB1 [28]. Here, we found that the protein expression levels of ZEB1, Snail, and SLUG were increased in the TMZresistant cells compared with the glioblastoma cells, indicating that EMT might contribute to the TMZ resistance of the glioblastoma cell line. Research has shown that cells with EMT characteristics tend to be more resistant to anticancer drugs and that the alteration of 
EMT status may affect the drug sensitivity of cancer cells. Similarly, the acquisition of an EMT phenotype can be observed in various drug-resistant cancer cells [29]. Some possible molecules or pathways that link EMT and MDR have been discussed, including the typical MDR-associated molecules, EMT regulators, cancer stem cells, and non-coding RNAs [30]. Our results confirmed that MALAT1 downregulation decreased ZEB1 protein expression but had no effect on Snail and SLUG protein expression. Further experiments suggested that MALAT1 most likely contributed to the regulation of TMZ resistance of glioblastoma cell lines by altering EMT status, specifically ZEB1 expression. A newly published paper established that MALAT1 functioned as a regulator in MDR through the HIF-2 $\alpha$-MALAT1-miR-216b axis via modulating autophagy in hepatocellular carcinoma cells [31], hinting at another possible pathway to influence MDR in cancer cells. However, the precise mechanisms of the role of MALAT1 in MDR should be further clarified.

In conclusion, the main findings of this study are that lncRNA MALAT1 functioned as a suppressor in the sensitivity of resistant glioblastoma cell lines to TMZ. Downregulation of MALAT1 reduced the TMZ resistance of the U251 and U87 cell lines by regulating ZEB1 expression. The present study offers a new insight for clarifying the potential mechanism of EMT in altering MDR in glioblastoma cells. MALAT1 is a potential target for developing new therapeutic strategies against drug resistance.

\section{Funding}

This study was supported by the National Natural Science Foundation of China (U1504819), the Foundation for Innovative Research Groups of the First Affiliated Hospital of Zhengzhou University (TD2011023) and the Scientific and Technological Project of Henan Province (112102310173).

\section{Disclosure Statement}

None.

\section{References}

1 Markman JL, Rekechenetskiy A, Holler E, Ljubimova JY: Nanomedicine therapeutic approaches to overcome cancer drug resistance. Adv Drug Deliv Rev 2013;65:1866-1879.

2 Messaoudi K, Clavreul A, Lagarce F: Toward an effective strategy in glioblastoma treatment. Part I: Resistance mechanisms and strategies to overcome resistance of glioblastoma to temozolomide. Drug Discov Today 2015;20:899-905.

3 Wick W, Platten M: Understanding and targeting alkylator resistance in glioblastoma. Cancer Discov 2014;4:1120-1122.

4 Kuwano M, Toh S, Uchiumi T, Takano H, Kohno K, Wada M: Multidrug resistance-associated protein subfamily transporters and drug resistance. Anticancer Drug Des 1999;14:123-131.

-5 Kalluri R, Weinberg RA: The basics of epithelial-mesenchymal transition. J Clin Invest 2009;119:14201428.

-6 Mallini P, Lennard T, Kirby J, Meeson A: Epithelial-to-mesenchymal transition: What is the impact on breast cancer stem cells and drug resistance. Cancer Treat Rev 2014;40:341-348.

7 Wang Z, Li Y, Ahmad A, Azmi AS, Kong D, Banerjee S, Sarkar FH: Targeting mirnas involved in cancer stem cell and emt regulation: An emerging concept in overcoming drug resistance. Drug Resist Update 2010;13:109-118.

-8 Moran VA, Perera RJ, Khalil AM: Emerging functional and mechanistic paradigms of mammalian long noncoding rnas. Nucleic acids Res 2012;40:6391-6400.

-9 Wapinski 0, Chang HY: Long noncoding rnas and human disease. Trends Cell Biol 2011;21:354-361. 


\section{Cellular Physiology Cell Physiol Biochem 2017;42:1192-1201

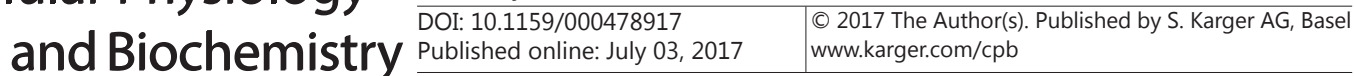

Li et al.: The Role of MALAT1 in the MDR of Glioblastoma

10 Prensner JR, Chinnaiyan AM: The emergence of Incrnas in cancer biology. Cancer Discov 2011;1:391-407.

11 Li C, Chen J, Zhang K, Feng B, Wang R, Chen L: Progress and prospects of long noncoding rnas (lncrnas) in hepatocellular carcinoma. Cellular Physiol Biochem 2015;36:423-434.

$\$ 12$ Okugawa Y, Toiyama Y, Hur K, Toden S, Saigusa S, Inoue Y, Mohri Y, Kusunoki M, Boland CR, Goel A: Metastasis-associated long non-coding rna drives gastric cancer development and promotes peritoneal metastasis. Carcinogenesis 2014:bgu200.

13 Jiang Y, Li Y, Fang S, Jiang B, Qin C, Xie P, Zhou G, Li G: The role of malat1 correlates with hpv in cervical cancer. Oncol Lett 2014;7:2135-2141.

14 Hirata H, Hinoda Y, Shahryari V, Deng G, Nakajima K, Tabatabai ZL, Ishii N, Dahiya R: Long noncoding rna malat1 promotes aggressive renal cell carcinoma through ezh2 and interacts with mir-205. Cancer Res 2015;75:1322-1331.

15 Ma KX, Wang HJ, Li XR, Li T, Su G, Yang P, Wu JW: Long noncoding rna malat1 associates with the malignant status and poor prognosis in glioma. Tumour Biol 2015;36:3355-3359.

16 Xiang J, Guo S, Jiang S, Xu Y, Li J, Li L, Xiang J: Silencing of long non-coding rna malat1 promotes apoptosis of glioma cells. J Korean Med Sci 2016;31:688-694.

17 Huarte M: The emerging role of Incrnas in cancer. Nat Med 2015;21:1253-1261.

18 Wang Y, Wu K, Yang Z, Zhao Q, Fan D, Xu P, Nie Y, Fan D: Multidrug-resistance related long non-coding rna expression profile analysis of gastric cancer. PLoS One 2015;10:e0135461.

19 Jiang M, Huang O, Xie Z, Wu S, Zhang X, Shen A, Liu H, Chen X, Wu J, Lou Y: A novel long non-coding rna-ara: Adriamycin resistance associated. Biochem Pharmacol 2014;87:254-283.

20 Gutschner T, Hammerle M, Diederichs S: Malat1 -- a paradigm for long noncoding rna function in cancer. J Mol Med (Berl) 2013;91:791-801.

21 Chufan EE, Sim HM, Ambudkar SV: Molecular basis of the polyspecificity of p-glycoprotein (abcb1): Recent biochemical and structural studies. Adv Cancer Res 2015;125:71-96.

-22 de Cremoux P, Jourdan-Da-Silva N, Couturier J, Tran-Perennou C, Schleiermacher G, Fehlbaum P, Doz F, Mosseri V, Delattre O, Klijanienko J, Vielh P, Michon J: Role of chemotherapy resistance genes in outcome of neuroblastoma. Pediatr Blood Cancer 2007;48:311-317.

23 Pan JJ, Xie XJ, Li X, Chen W: Long non-coding rnas and drug resistance. Asian Pac J Cancer Prev 2015;16:8067-8073.

24 Yang Y, Li H, Hou S, Hu B, Liu J, Wang J: The noncoding rna expression profile and the effect of lncrna ak126698 on cisplatin resistance in non-small-cell lung cancer cell. PLoS One 2013;8:e65309.

25 Liu Z, Sun M, Lu K, Liu J, Zhang M, Wu W, De W, Wang Z, Wang R: The long noncoding rna hotair contributes to cisplatin resistance of human lung adenocarcinoma cells via downregualtion of p21(waf1/cip1) expression. PLoS One 2013;8:e77293.

-26 Tsang WP, Kwok TT: Riboregulator h19 induction of mdr1-associated drug resistance in human hepatocellular carcinoma cells. Oncogene 2007;26:4877-4881.

27 Yang J, Weinberg RA: Epithelial-mesenchymal transition: At the crossroads of development and tumor metastasis. Dev cell 2008;14:818-829.

-28 Schulte J, Weidig M, Balzer P, Richter P, Franz M, Junker K, Gajda M, Friedrich K, Wunderlich H, Ostman A, Petersen I, Berndt A: Expression of the e-cadherin repressors snail, slug and zeb1 in urothelial carcinoma of the urinary bladder: Relation to stromal fibroblast activation and invasive behaviour of carcinoma cells. Histochem Cell Biol 2012;138:847-860.

29 Wang Z, Li Y, Kong D, Banerjee S, Ahmad A, Azmi AS, Ali S, Abbruzzese JL, Gallick GE, Sarkar FH: Acquisition of epithelial-mesenchymal transition phenotype of gemcitabine-resistant pancreatic cancer cells is linked with activation of the notch signaling pathway. Cancer Res 2009;69:2400-2407.

-30 Shang Y, Cai X, Fan D: Roles of epithelial-mesenchymal transition in cancer drug resistance. Curr Cancer Drug Targets 2013;13:915-929.

-31 Yuan P, Cao W, Zang Q, Li G, Guo X, Fan J: The hif-2 $\alpha$-malat1-mir-216b axis regulates multi-drug resistance of hepatocellular carcinoma cells via modulating autophagy. Biochem Biophys Res Commun 2016;478:1067. 